\title{
Real-time calibration and alignment of the LHCb RICH detectors
}

\author{
Jibo HE \\ University of Chinese Academy of Sciences (UCAS), Beijing, China
}

\section{A R T I C L E I N F O}

On behalf of the LHCb RICH collaboration

Keywords:

$\mathrm{RICH}$

Cherenkov detectors

Calibration

Alignment

$\mathrm{LHCb}$

\begin{abstract}
A B S T R A C T
In 2015, the LHCb experiment established a new and unique software trigger strategy with the purpose of increasing the purity of the signal events by applying the same algorithms online and offline. To achieve this, real-time calibration and alignment of all LHCb sub-systems is needed to provide vertexing, tracking, and particle identification of the best possible quality. The calibration of the refractive index of the RICH radiators, the calibration of the Hybrid Photon Detector image, and the alignment of the RICH mirror system, are reported in this contribution. The stability of the RICH performance and the particle identification performance are also discussed.
\end{abstract}

\section{Introduction}

The LHCb experiment [1] is one of the four large particle physics experiments at the Large Hadron Collider (LHC), and is designed to search for physics beyond the Standard Model by precision study of the beauty and charm hadrons. The Ring Imaging CHerenkov (RICH) detectors of the LHCb experiment employ the $\mathrm{C}_{4} \mathrm{~F}_{10}$ and $\mathrm{CF}_{4}$ radiators to provide particle identification (PID) of charged particles in the momentum range of $2-100 \mathrm{GeV}$. This is essential for the $\mathrm{LHCb}$ core physics program. The centre-of-mass energy of the LHC has been increased from $8 \mathrm{TeV}$ to $13 \mathrm{TeV}$ in 2015, and the LHCb experiment will be upgraded [2,3], starting in 2019, and run at a 4-times higher luminosity than in LHC Runs I and II. A new and unique software trigger strategy has been established at the LHCb experiment with the purpose of increasing the purity of the signal events by applying the same algorithms online and offline. This requires ultimate quality of vertexing, tracking, and PID. Therefore, real-time calibration and alignment of all LHCb sub-systems is required online.

The calibration of the refractive index of the RICH radiators, the calibration of the Hybrid Photon Detector (HPD) image, and the alignment of the RICH mirror system are reported here. The LHCb trigger strategy in Run-II (2015-2018) has been changed with respect to that in Run-I (2010-2013), as shown in Fig. 1. The online event reconstruction in Run-I was simpler and faster than that used offline, and did not have the latest detector calibration and alignment constants applied. In the Run-II data-taking, the events selected by the first stage of the software trigger are buffered on local disks, then an automatic detector calibration and alignment is performed, and the resulting calibration and alignment constants are used in the final stage of the software trigger. As the full offline event reconstruction is run in the final stage of the software trigger, its output can be used for physics analysis directly without further offline processing.

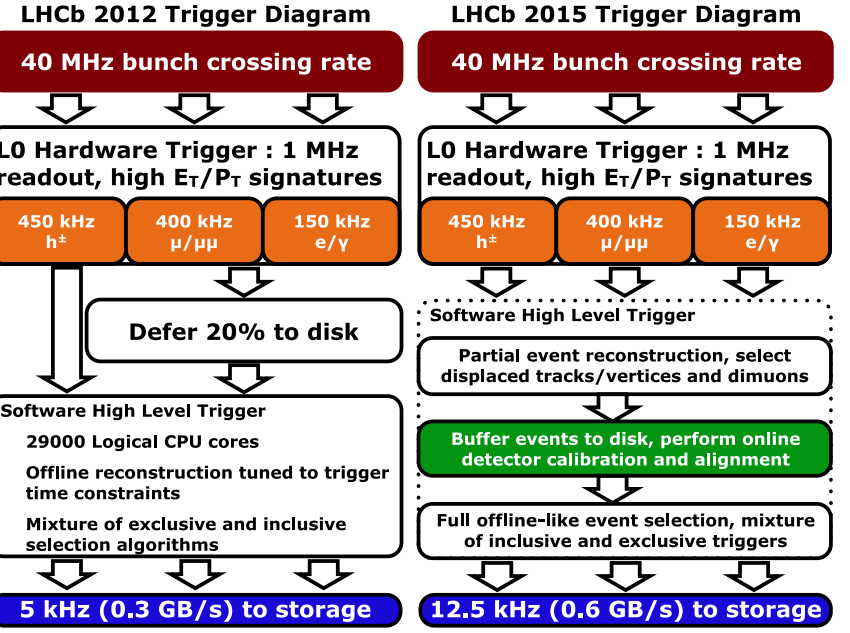

Fig. 1. Schematic diagram of the LHCb trigger data-flow in Run-I data taking (left) compared to the data-flow in Run-II (right).

\section{RICH optical layout and reconstruction}

The LHCb RICH system has two detectors, one upstream and one downstream of the magnet, covering the low momentum range $\sim 2-$ $60 \mathrm{GeV} / \mathrm{c}$ and the high momentum range $\sim 15-100 \mathrm{GeV} / \mathrm{c}$ respectively [1]. The side view of the RICH detector upstream of the magnet is shown in Fig. 2. The Cherenkov photons emitted by charged particle tracks in the radiator are reflected and focused by the combination of spherical and secondary mirrors out of the LHCb geometrical acceptance and then are detected by the Hybrid Photon Detectors (HPD).

To reconstruct the photon candidate and the Cherenkov angle, one 


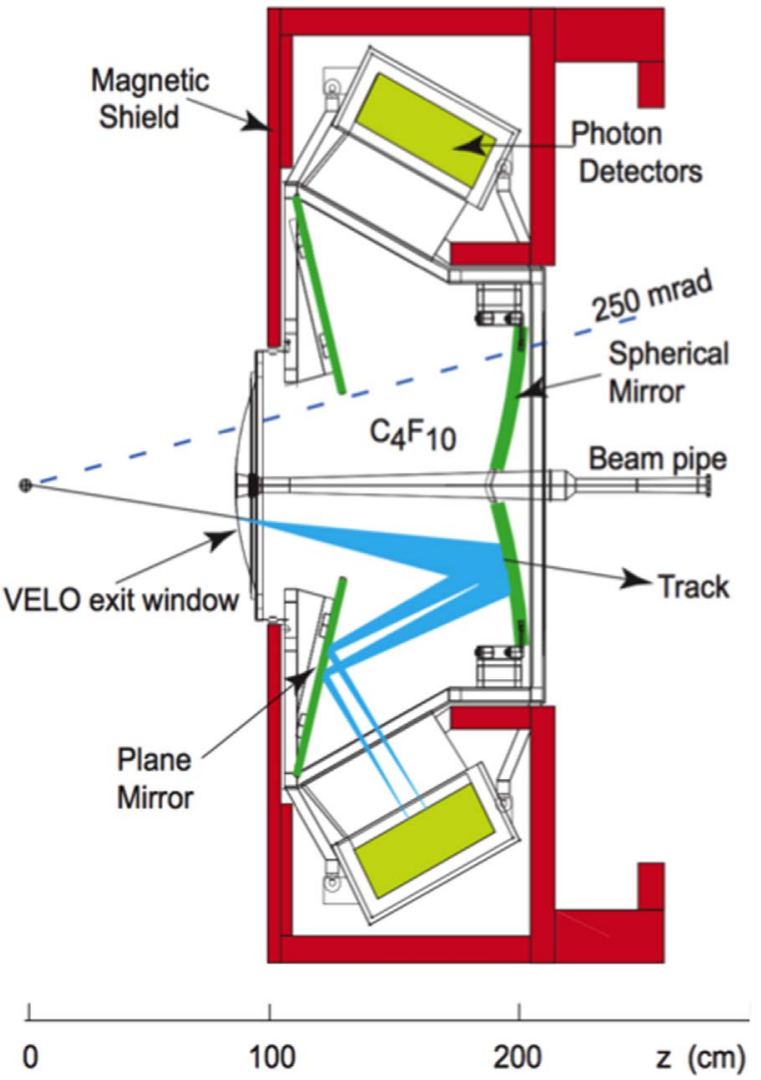

Fig. 2. Side view of the LHCb RICH detector upstream of the magnet.

takes the spatial position of the HPD pixel hits as the detection point, and the middle point of the associated track in the radiator as the emission point, then solves a quartic equation that fully describes the reflections of the photon, given the RICH geometry [4]. The "global likelihood algorithm" [5] is used to determine the PID, where information from all tracks, all radiators and all pixel hits within a given event are considered simultaneously, and the likelihood is maximized by comparing the observed Cherenkov angles with those expected under different PID hypotheses.

To achieve the best PID performance, one needs to align the RICH mirrors, detector planes and the tracking system, and calibrate the refractive index of radiators and the HPD image with good precision. These factors are all time-dependent, necessitating real-time calibration and alignment of the LHCb RICH detectors, and the tracking system.

\section{Calibration and alignment}

\subsection{Calibration of the refractive index of the $\mathrm{RICH}$ radiators}

The refractive index of the gas radiators depends on the ambient temperature and pressure, and the exact composition of the gas mixture; so it can change in time. These quantities are monitored by hardware to compute an expected refractive index, but this does not have a precision that is high enough for the physics analysis, therefore it needs to be further corrected. As shown in Fig. 3, the distribution of the difference between the reconstructed and expected Cherenkov angle is fitted to obtain the shift, which is then converted to a scale factor of the expected refractive index according to studies based on simulation.

About $50 \mathrm{~Hz}$ of events are sent to multiple online reconstruction tasks, which run in parallel, and the resulting histograms are merged at

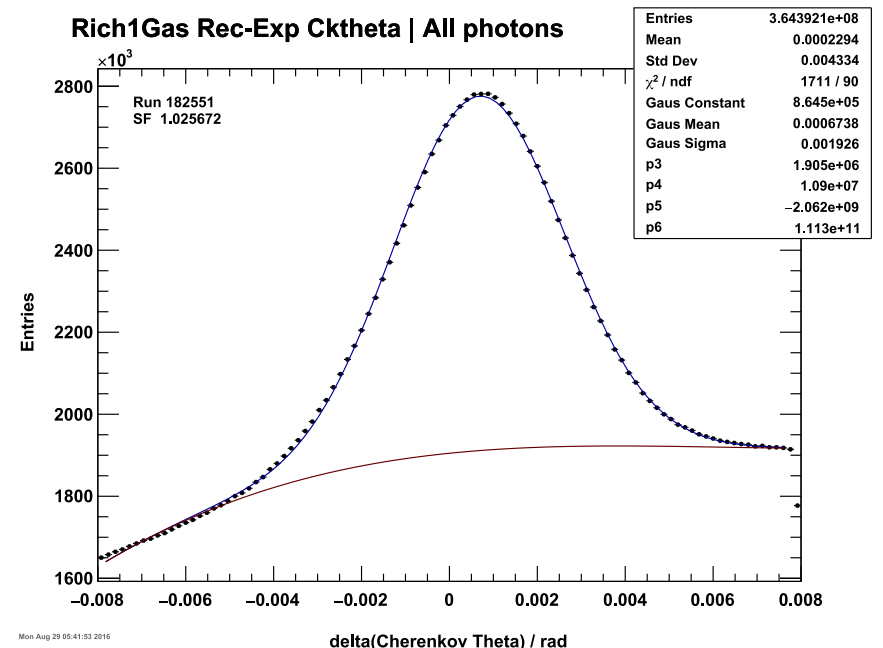

Fig. 3. Difference between the reconstructed and expected Cherenkov angle before the calibration. The mean value, determined from the fit shown, is then converted to a correction factor that is applied to the expected refractive index.

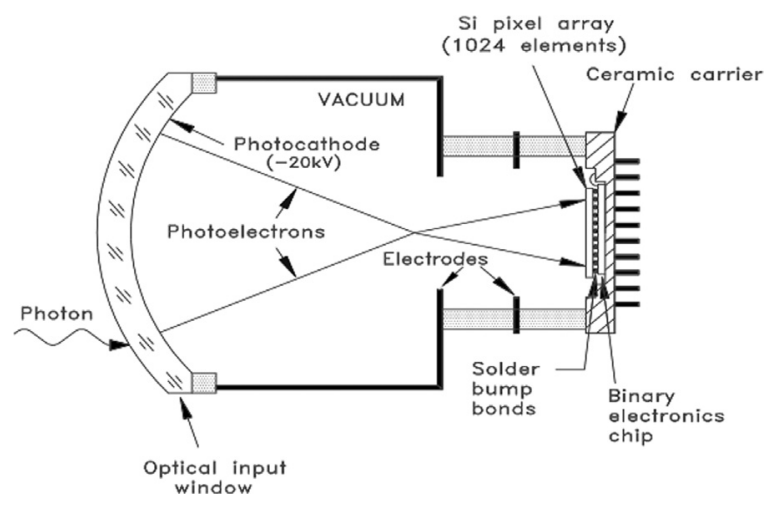

Fig. 4. Schematic drawing of the Hybrid Photon Detector (HPD).

the end of each run. Then a dedicated task is used to fit the histograms merged run-by-run ${ }^{1}$ and produce calibration constants to be used by the RICH reconstruction in the final stage of the software trigger.

\subsection{Calibration of the HPD images}

The Hybrid Photon Detector is used to detect Cherenkov photons. As shown in Fig. 4, the photoelectron produced at the photocathode is accelerated by a high voltage of up to $20 \mathrm{kV}$ onto a reverse-biased pixellated silicon detector, with a de-magnification factor of about 5 [6]. The HPD anode images are affected by the magnetic and electric fields, and have been observed to move and change their size, possibly due to changes in these residual fields when the high voltage is cycled each LHC fill. Such changes could degrade the reconstruction of the Cherenkov angle and affect the PID performance. Therefore the centre and radius of all the HPD images are calibrated run-by-run. Fig. 5 shows the calibration process. First, the centre of the image is cleaned to eliminate ion feedback. Then a Sobel filter is used to detect the edges of the image that are fitted to determine the centre and the radius of the image, which are used by the RICH reconstruction in the final stage of the software trigger. As only the raw HPD data needs to be decoded, more than $500 \mathrm{~Hz}$ of events are processed run-by-run.

\footnotetext{
${ }^{1}$ The maximum run length is one hour
} 
Rich_HPD_133_Image

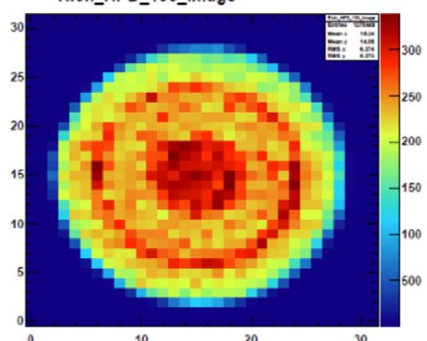

(a)

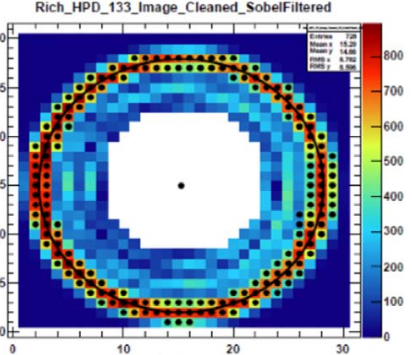

(c)

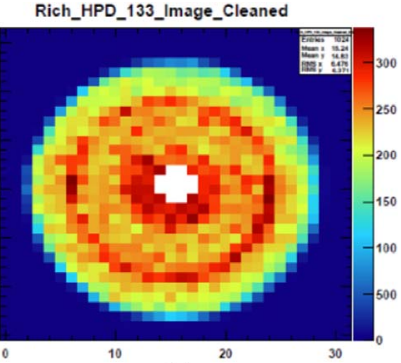

(b)

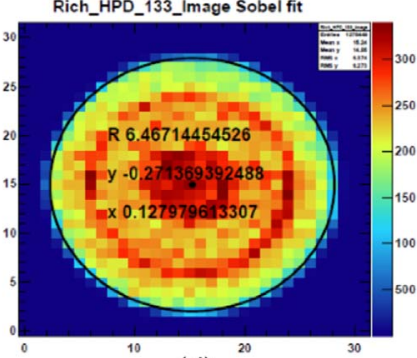

(d)
Fig. 5. Calibration process of the HPD image: (a) HPD image for a typical run. (b) The centre of the HPD image is cleaned to eliminate ion feedback. (c) Edge of the HPD image detected by the Sobel filter. (d) Radius and centre of the HPD image returned by the fit.
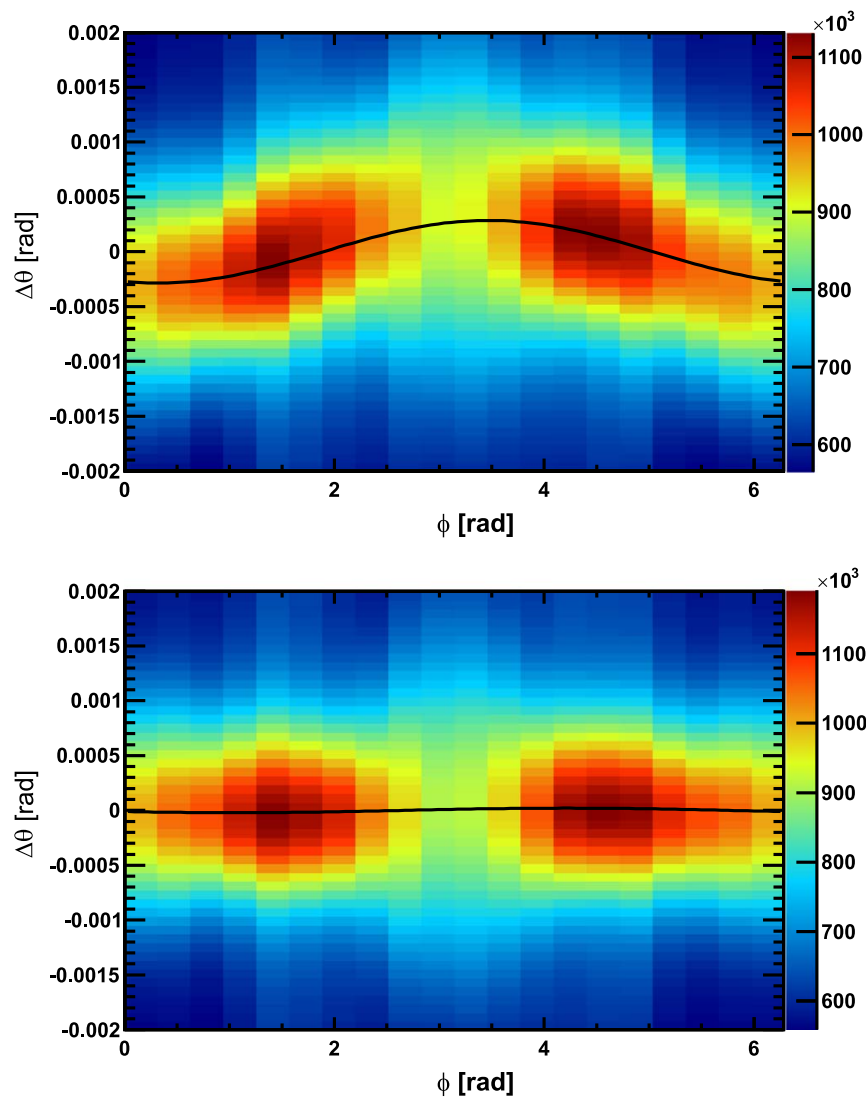

Fig. 6. Difference between the measured and expected Cherenkov angle, $\Delta \theta_{C}$ plotted as a function of the azimuthal angle $\phi$ and fitted with $\theta_{x} \cos (\phi)+\theta_{y} \sin (\phi)$, for one side of the RICH 2 detector [6]. The upper plot is prior to alignment, and shows a dependency of the angle $\theta_{C}$ on the angle $\phi$. The bottom plot is after the alignment correction, and $\Delta \theta_{C}$ is uniform in $\phi$.
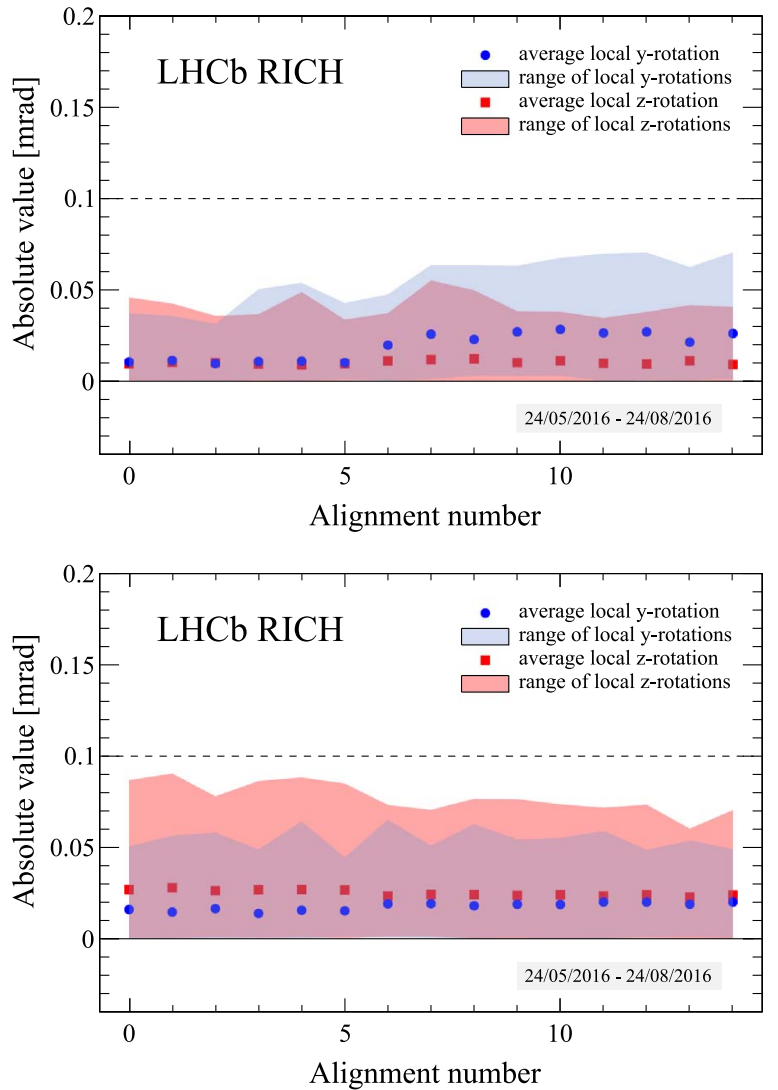

Fig. 7. Time dependence of the mirror alignment parameters for the RICH detector downstream the magnet for the 2016 data sample, (upper) spherical mirror, (bottom) secondary mirror. The shadow regions show the range of alignment parameters for all the mirror pairs, the points show the average value for all the mirror pairs.

\subsection{Alignment of the RICH mirror system}

The Cherenkov photons emitted by the charged particles passing through the RICH detectors are focused onto the photon-detector plane by the spherical and secondary mirrors. In case of misalignment the centre of Cherenkov ring would not correspond to the intersection point of the charged track, and this would introduce a dependence of the difference between the measured and expected Cherenkov angle on the azimuthal angle of the ring, as shown in Fig. 6. The alignment constants for each mirror are determined by the fit of the Cherenkov angle difference as a function of the azimuthal angle on the ring. The correlation between the different mirror pairs is also taken into account. The procedure is evaluated by an iterative procedure implemented in a dedicated framework, which makes it possible to run the alignment in parallel using about 1800 nodes of the software trigger farm. The alignment of the RICH mirror system has been found to be stable enough to not affect the PID performance, as shown in Fig. 7 , and it runs routinely as a monitoring task.

\subsection{Time alignment}

In order to maximise the photon collection efficiency of the LHCb RICH detectors, the HPD readout must be synchronised with the LHC bunch crossing to within a few nanoseconds. The initial time alignment was performed in the absence of beam using a pulsed laser, and has been improved further with dedicated timing scan data taken during physics collisions. As shown in Fig. 8, all the HPDs have been time aligned to about $1 \mathrm{~ns}$. 

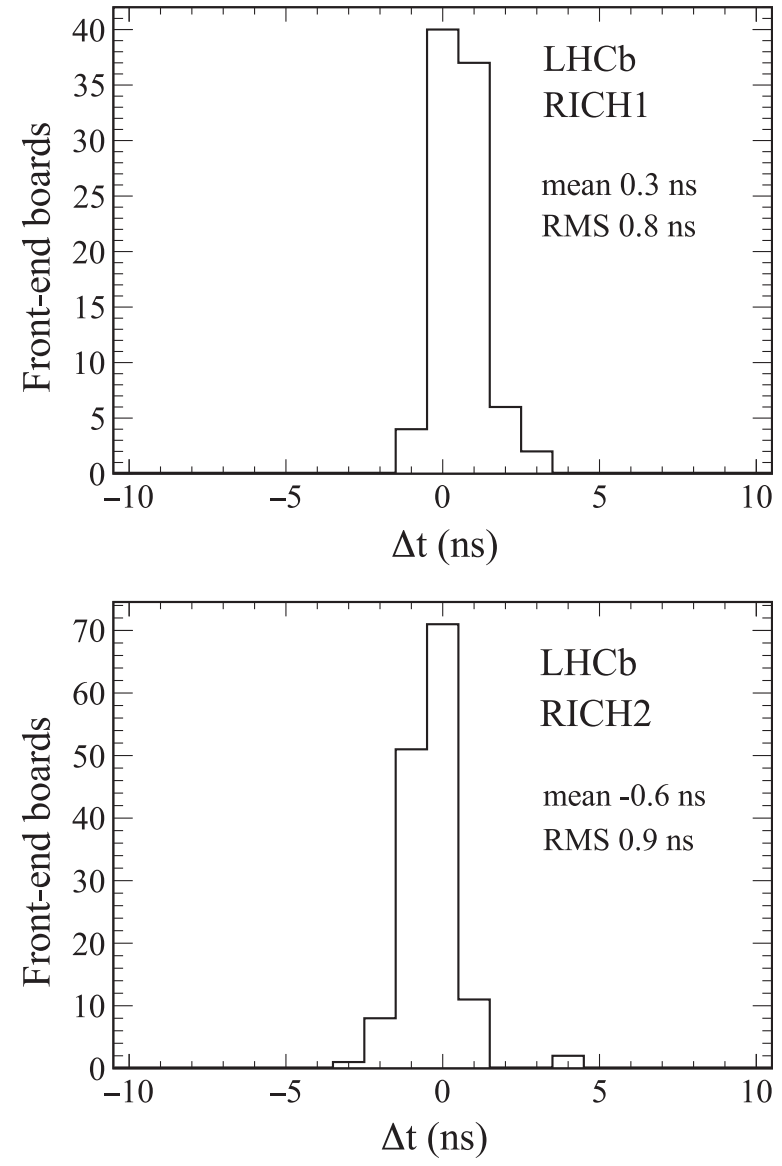

Fig. 8. Distribution of the midpoints of timing scans in the upstream RICH (upper) and downstream RICH (bottom) after time alignment with pp collisions.

\section{Performance}

\subsection{Stability of the RICH performance}

The stability of the RICH performance is monitored by the Cherenkov angle resolution obtained with the same RICH mirror

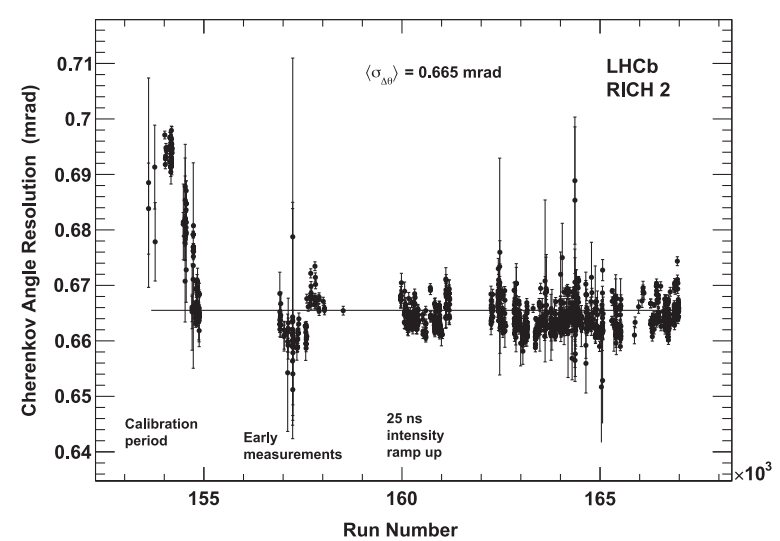

Fig. 9. Time dependence of the Cherenkov angle resolution for the RICH detector downstream of the magnet for the 2015 data sample. alignment and with the dedicated run-by-run calibrations. It has been found to be stable for the full 2015 data-taking, as shown in Fig. 9.

\subsection{Particle identification performance}

Fig. 10 shows the kaon efficiency (kaons identified as kaons) and pion misidentification (pions misidentified as kaons), as a function of particle momentum, obtained from imposing two different requirements on this distribution in the Run-II data. One can see that the RICH detectors provide excellent particle identification. Further details on the particle identification performance in the Run-II data-taking of the LHCb experiment can be found in Ref. [7].

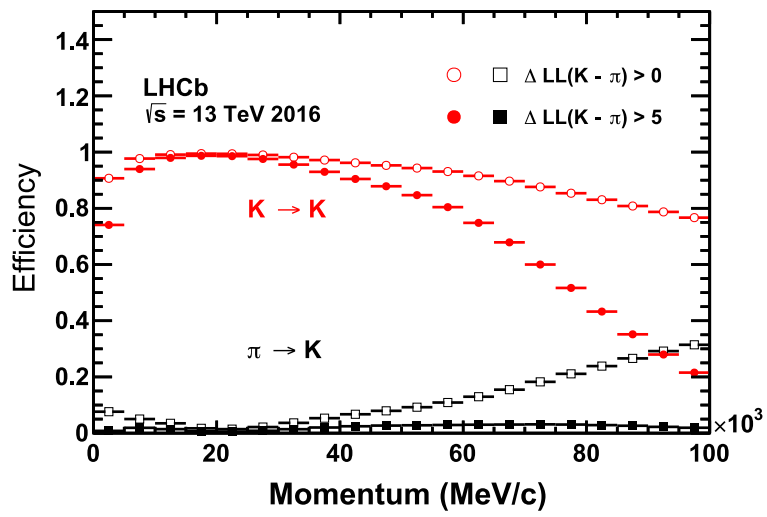

Fig. 10. Kaon identification efficiency and pion misidentification rate measured on data as a function of track momentum. Two different $\Delta \log \mathcal{L}(K-\pi)$ requirements have been imposed on the samples, resulting in the open and filled marker distributions, respectively.

\section{Summary}

Novel real-time calibration and alignment of the $\mathrm{LHCb} \mathrm{RICH}$ detectors have been implemented in the Run-II data-taking of the $\mathrm{LHCb}$ experiment. This includes the run-by-run calibration of the refractive index of the RICH radiators, and that of the Hybrid Photon Detector image, and the alignment of the RICH mirror system. The real-time calibration and alignment works well, and provides excellent particle identification for the online trigger and offline analysis.

\section{References}

[1] A.A. Alves, et al., LHCb collaborationThe LHCb Detector at the LHC, , J. Instrum. 3 (2008) S08005.

[2] LHCb collaboration, Framework TDR for the LHCb Upgrade: Technical Design Report, CERN-LHCC-2012-007.

[3] LHCb collaboration, LHCb PID Upgrade Technical Design Report, CERN-LHCC2013-022.

[4] T. Ypsilantis, J. Seguinot, Theory of ring imaging Cherenkov counters, Nucl. Instrum. Methods Phys. Res. A 343 (1994) 30.

[5] R. Forty, RICH pattern recognition for LHCb, Nucl. Instrum. Methods Phys. Res. A 433 (1999) 257.

[6] M. Adinolfi, et al., Performance of the LHCb RICH detector at the LHC, Eur. Phys. J. C. 73 (2013) 2431 (http://arXiv:1211.6759arXiv:1211.6759).

[7] A. Papanestis, Performance of the LHCb RICH detectors during the LHC Run II, these proceedings. 